NONCOMMUTATIVE GEOMETRY

AND QUANTUM GROUPS

BANACH CENTER PUBLICATIONS, VOLUME 61

INSTITUTE OF MATHEMATICS

POLISH ACADEMY OF SCIENCES

WARSZAWA 2003

\title{
A NEW CONSTRUCTION OF CHARACTERISTIC CLASSES FOR NONCOMMUTATIVE ALGEBRAIC PRINCIPAL BUNDLES
}

\author{
GEORGIY I. SHARYGIN \\ Group of Mathematics, ITEP \\ B. Cheremushkinskaia ul., 25, 117218 Moscow, Russia \\ E-mail: sharygin@gate.itep.ru
}

\begin{abstract}
We propose a new construction of characteristic classes for noncommutative algebraic principal bundles (Hopf-Galois extensions) with values in Hochschild and cyclic homology.

1. Introduction and preliminaries. Our work is primarily based on the ideas M. Đurđevič [6]-[8]. The purpose of this paper is to give a unifying construction of characteristic classes generalizing the approaches of [7, 8] and [12]. We begin with the definitions of Hopf-Galois extensions, connections on them and curvatures. All the algebras we consider below are unital and over the field of complex numbers, though most of the results discussed below can be formulated for an arbitrary commutative ground ring. We use the standard notation for coaction and coproducts.
\end{abstract}

Definition 1. Let $\mathcal{H}$ be a Hopf algebra and $\mathcal{B}$ a right $\mathcal{H}$-comodule algebra via a coaction $F: \mathcal{B} \rightarrow \mathcal{B} \otimes \mathcal{H}$. We call $\mathcal{B}$ a noncommutative algebraic principal bundle if the map

$$
\chi: \mathcal{B} \otimes \mathcal{B} \rightarrow \mathcal{B} \otimes \mathcal{H}, \quad b \otimes b^{\prime} \mapsto b F\left(b^{\prime}\right),
$$

is surjective. The Hopf algebra $\mathcal{H}$ is referred to as the quantum structure group, and the algebra of coinvariants

$$
\mathcal{M} \stackrel{\text { def }}{=} \mathcal{B}^{\text {Co } \mathcal{H}}:=\{b \in \mathcal{B} \mid F(b)=b \otimes 1\}
$$

as the base of this bundle.

The first occurrence of this definition (in a purely algebraic context) was in [11], where such an object is called a "Hopf-Galois extension" (of the algebra $\mathcal{M}$ by the Hopf algebra $\mathcal{H}$ ). With time, this definition was modified to assume the following form:

2000 Mathematics Subject Classification: Primary 58B34.

The paper is in final form and no version of it will be published elsewhere.

The author was partially supported by the grant RFFI 01-01-0546 
Definition 2. A noncommutative algebraic principal bundle $\mathcal{B}$ is a Hopf-Galois extension if the induced map

$$
\tilde{\chi}: \mathcal{B} \otimes_{\mathcal{M}} \mathcal{B} \rightarrow \mathcal{B} \otimes \mathcal{H}, \quad b \otimes_{\mathcal{M}} b^{\prime} \mapsto b F\left(b^{\prime}\right) .
$$

is bijective.

As H.-J. Schneider explained in his lectures, if $\mathcal{B}$ is $\mathcal{H}$-coflat, these two definitions are equivalent. As there appear to be no interesting examples of noncommutative algebraic bundles with non-injective canonical map $\tilde{\chi}$, we shall from now on assume the injectivity of $\tilde{\chi}$ and use the expressions "noncommutative algebraic principal bundle" and "HopfGalois extension" interchangeably.

The notion of a Hopf-Galois extension was first treated by the methods of noncommutative geometry in two independent papers: one of T. Brzeziński and S. Majid [1], and the other one of M. Đurđevič [7]. The former adopts the new and the latter the old definition of a Hopf-Galois extension. The main difference of the two approaches emerges in the definitions of differential structures on such bundles. Since Đurđevič's approach seems to be less restrictive as far as the higher-order differential forms are concerned, we follow it herein.

Let $\Omega(\mathcal{H}$ ) be a bicovariant differential calculus on the Hopf algebra $\mathcal{H}$ (see $[6,7]$ and [14] for definitions). ${ }^{1}$ Assume that the bialgebra structure on $\mathcal{H}$ (i.e. the comultiplication and counit but not necessarily the antipode) can be extended to $\Omega(\mathcal{H})$. For instance, this is always the case if one considers the "universal differential envelope" of the first order bicovariant differential calculus (see, e.g., [6], App. A).

Definition 3. A differential graded algebra $\Omega(\mathcal{B})$ is a differential calculus on the noncommutative algebraic principal bundle $\mathcal{B}$ corresponding to the differential calculus $\Omega(\mathcal{H})$ if

(i) $\Omega(\mathcal{B})$ is generated by $\Omega_{0}(\mathcal{B})=\mathcal{B}$ as a differential graded algebra;

(ii) $\Omega(\mathcal{B})$ is a right $\Omega(\mathcal{H})$-comodule algebra.

Note that these algebras are to be thought of as differential graded algebras, so that on the 0 -th degree elements this comodule structure coincides with $F$.

We shall denote the corresponding $\Omega(\mathcal{H})$-comodule algebra structure by $\widehat{F}: \Omega(\mathcal{B}) \rightarrow$ $\Omega(\mathcal{B}) \otimes \Omega(\mathcal{H})$. Here $\otimes$ signifies the tensor product in the graded category, i.e., for two graded algebras $A$ and $B$ one puts

$$
\begin{gathered}
(A \otimes B)_{n}=\bigoplus_{i+j=n} A_{i} \otimes B_{j}, \\
\left(a_{1} \otimes b_{1}\right)\left(a_{2} \otimes b_{2}\right)=(-1)^{\left|b_{1}\right|\left|a_{2}\right|} a_{1} a_{2} \otimes b_{1} b_{2},
\end{gathered}
$$

and the differential is determined by the graded Leibniz rule. Observe that, if the map $\widehat{F}$ exists, one can define it in the following way. On $\Omega_{1}(B)$ put

$$
\widehat{F}(a d b)=\sum a_{(0)} d b_{(0)} \otimes a_{(1)} b_{(1)}+a_{(0)} b_{(0)} \otimes a_{(1)} d b_{(1)},
$$

\footnotetext{
${ }^{1}$ In this paper we use the expression "differential calculus" when speaking about the whole algebra of differential forms, as in [7], and not just about its first order part, as in [14].
} 
(the differential on the right term is in $\Omega(\mathcal{H})$ ), and then extend it to the higher degree forms in the obvious way to obtain a homomorphism.

Note that such differential calculi on $\mathcal{B}$ always exist. In fact, it is always possible to choose, for instance, the universal differential calculi on this algebra and $\mathcal{H}$ (see [10], [4], $\S 1$ or [6], App. A for the definition of the universal calculus). Then, by virtue of its universality, the formula (4) gives a well-defined morphism of the modules of 1-forms and can be extended to the whole $\Omega(\mathcal{B})$. (Recall, that the universal differential calculus on an algebra $A$ is completely characterized by the following property: for any algebra homomorphism $f: A \rightarrow \Omega$ from $A$ to an arbitrary differential algebra $\Omega$, there exists a unique homomorphism $f^{*}: \Omega(A) \rightarrow \Omega$ of differential graded algebras extending $f$.)

Much as before, one puts

$$
\Omega(\mathcal{M}) \stackrel{\text { def }}{=} \Omega(\mathcal{B})^{\operatorname{Co} \Omega(\mathcal{H})} .
$$

Clearly, $\Omega(\mathcal{M})$ is a differential graded subalgebra in $\Omega(\mathcal{B})$ containing $\mathcal{M}$. In addition, one defines the subalgebra of horizontal forms as

$$
\mathfrak{h o r}(\mathcal{B}) \stackrel{\text { def }}{=} \widehat{F}^{-1}(\Omega(\mathcal{B}) \otimes \mathcal{H}) .
$$

Notice that $\Omega(\mathcal{M}) \subseteq \mathfrak{h o r}(\mathcal{B})$ though in general $d \mathfrak{h o r}(\mathcal{B}) \not \subset \mathfrak{h o r}(\mathcal{B})$. One can also consider the right $\mathcal{H}$-comodule algebra structure on $\Omega(\mathcal{B})$ by composing $\widehat{F}$ with the natural projection on the degree 0 component of $\Omega(\mathcal{H})$ in this tensor product. This coaction will be denoted by $F^{\wedge}$.

Now we can proceed to M. Đurđevič's definitions of connections and curvatures. From now on, for the sake of notational simplicity, we suppose that the bicovariant differential calculus on $\mathcal{H}$ is the universal one. (The general case can be treated analogously, see [7].) Then the space of left-invariant differential 1-forms on $\mathcal{H}$ (denoted by $\Gamma_{\text {inv }}$ ) is isomorphic to ker $\epsilon$. Here $\epsilon$ is the counit of the Hopf algebra $\mathcal{H}$. The isomorphism is given by:

$$
\pi(h)=\sum S\left(h_{(1)}\right) d h_{(2)}, \forall h \in \operatorname{ker} \epsilon .
$$

Moreover, the space of left-invariant $n$-forms is isomorphic to $\Gamma_{i n v}^{\otimes n}=\operatorname{ker} \epsilon^{\otimes n}$. Let $\varpi$ (resp. $\varpi^{\otimes n}$ ) denote the restriction of the right $\mathcal{H}$-coaction on $\Omega(\mathcal{H})$ to $\Gamma_{\text {inv }}$ (resp. to $\left.\Gamma_{i n v}^{\otimes n}\right)$. Note that under the isomorphism $\pi^{-1}: \Gamma_{i n v} \cong \operatorname{ker} \epsilon$ this restriction coincides with the restriction to ker $\epsilon$ of the right adjoint coaction $a d: \mathcal{H} \rightarrow \mathcal{H} \otimes \mathcal{H}$ of $\mathcal{H}$ on itself:

$$
a d(h)=\sum h_{(2)} \otimes S\left(h_{(1)}\right) h_{(3)}, \quad h \in \operatorname{ker} \epsilon .
$$

The following two definitions are taken from [7].

Definition 4. A linear map $\varphi: \Gamma_{\text {inv }} \rightarrow \Omega(\mathcal{B})$ is called a pseudotensorial form on $\mathcal{B}$ if it intertwines the coaction of $\mathcal{H}$ on both spaces ( $\varpi$ on the left and $F^{\wedge}$ on the right), i.e. if the following diagram commutes:

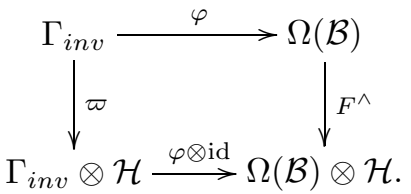

A pseudotensorial form $\varphi$ is called tensorial if the image of $\varphi$ is in $\mathfrak{h o r}(\mathcal{B})$. 
Let us remark that the spaces of pseudotensorial and tensorial forms on $\mathcal{B}$ are graded by the grading induced from $\Omega(\mathcal{B})$.

Definition 5. A pseudotensorial 1-form $\omega$ on $\mathcal{B}$ is called a connection (or connection 1-form) on the noncommutative algebraic principal bundle if

$$
\widehat{F}(\omega(\theta))=\sum \omega\left(\theta_{(0)}\right) \otimes \theta_{(1)}+1 \otimes \theta, \quad \sum \theta_{(0)} \otimes \theta_{(1)}:=\varpi(\theta) .
$$

Connections of this sort we shall sometime call "Đurđevič's connections" in order to distinguish them from those introduced by T. Brzeziński, S. Majid and others in their works on Hopf-Galois extensions.

Given a connection (its existence is proven in [7] and [13, Satz 6.3.5]) one can define, for any natural $n$, the following map:

$$
\begin{aligned}
m_{\omega}^{n}: \mathfrak{h o r}(\mathcal{B}) \otimes \Gamma_{i n v}^{\otimes n} & \rightarrow \Omega(\mathcal{B}), \\
h \otimes \theta_{1} \otimes \ldots \otimes \theta_{n} & \mapsto h \omega\left(\theta_{1}\right) \ldots \omega\left(\theta_{n}\right) .
\end{aligned}
$$

Here $h \in \mathfrak{h o r}(\mathcal{B}), \theta_{i} \in \Gamma_{i n v}, i=1, \ldots, n$. Now one can prove the following [7]:

THEOREM 1. The maps $m_{\omega}^{n}, n \in \mathbb{N}$, induce a right $\Omega(\mathcal{H})$-colinear isomorphism of graded spaces

$$
m_{\omega}: \bigoplus_{n \geq 0} \mathfrak{h o r}(\mathcal{B}) \otimes \Gamma_{i n v}^{\otimes n} \rightarrow \Omega(\mathcal{B}) .
$$

The grading on the left is given by the sum of gradings in the factors.

In general, this map is only an isomorphism of graded spaces with $\Omega(\mathcal{H})$-coaction, and respects neither the differential, nor the multiplication. While the former fact lies completely within the classical theory (it is equivalent to the observation that the square of the covariant differential, i.e., the curvature is not necessarily zero in general), the latter fact is a completely noncommutative phenomenon. To deal with this, the following notion was introduced by M. Đurđevič:

Definition 6. A connection $\omega$ is regular if the following equation holds:

$$
\omega(\pi(h)) \psi=(-1)^{|\psi|} \sum_{k} \psi_{k} \omega\left(\pi\left(h c_{k}\right)\right), \quad F^{\wedge}(\psi)=: \sum_{k} \psi_{k} \otimes c_{k},
$$

for any $h \in \operatorname{ker} \epsilon$ and $\psi \in \mathfrak{h o r}(\mathcal{B})$.

It turns out [7] that regular connections turn $m_{\omega}$ of (13) into an isomorphism of algebras. Now one can proceed in the usual way to construct Chern-Weil classes with values in the homology of $\Omega(\mathcal{M})$. We shall not reproduce this here, see [7] for details. However, we shall need an explicit formula for the curvature of a connection in the case of the universal differential calculus on the Hopf algebra $\mathcal{H}$ :

$$
R_{\omega}(\pi(h))=d \omega(\pi(h))-\omega\left(\pi\left(h_{(1)}\right)\right) \omega\left(\pi\left(h_{(2)}\right)\right), h \in \operatorname{ker} \epsilon .
$$

Let us now compare these notions and those appearing in [1], [5], [9], and many other papers. First, recall the definition of a connection 1-form on a noncommutative algebraic principal bundle given in the quoted papers. (We give here only the version dealing with the universal calculus. The general case is treated, e.g., in [1], [9].) 
Definition 7. A linear map $\omega: \operatorname{ker} \epsilon \rightarrow \Omega_{1}(\mathcal{B}):=\operatorname{ker}(m: \mathcal{B} \otimes \mathcal{B} \rightarrow \mathcal{B})$ is a connection 1-form (or, by abuse of language, connection) if it satisfies the following properties:

(i) $(m \otimes$ id $) \circ($ id $\otimes F) \circ \omega=1 \otimes i d$,

(ii) $\left(F^{\wedge}\right)_{\mid \Omega_{1}(\mathcal{B})} \circ \omega=(\omega \otimes$ id $) \circ$ ad.

A connection $\omega$ is called strong if

(iii) $d p-p_{(0)} \omega\left(p_{(1)}\right) \in\left(\Omega_{1}(\mathcal{M})\right) \mathcal{B}, \forall p \in \mathcal{B}$.

Note that in this definition we have used the notation introduced above, which does not coincide with that of the cited papers. For example, the map $(m \otimes \mathrm{id}) \circ(\mathrm{id} \otimes F)$ from the first part of Definition 7 is denoted therein by $\tilde{\varkappa}: \Omega_{1}(\mathcal{B}) \rightarrow \mathcal{B} \otimes \operatorname{ker} \epsilon$.

We shall not discuss this approach here in full detail, see the quoted papers for exact formulations. Let us just stress the main difference between the two approaches. Roughly speaking, in the Brzeziński-Majid approach, the (first order) differential forms on the base of a Hopf-Galois extension (coinvariant subalgebra) are generated by the base itself. In Đurđevič's theory the differential calculus on the base depends to a great extent on the structure of the "total space" $\mathcal{B}$. This evidently follows from the definitions given above.

However, this difference does not appear in Definition 7, and one can try to use both definitions at the same time. Then we have the following simple observation describing the relation between Đurđevič's connections and those of the last definition. (In a more or less explicit form it has already appeared in [7], Section 6.3.)

Proposition 2. For the universal differential calculi on $\mathcal{H}$ and $\mathcal{B}$, Durdevič's definition and Definition 7 are equivalent.

Proof. The proof relies on observing that the formulas used to define a connection form $\omega$ in Definition 7 are just extended (coordinate) versions of those listed in Đurđevič's definitions. To see it, just notice that the map $\tilde{\varkappa}$ from the first part of Definition 7 is the composition of $\widehat{F}$, projection on the component $\mathcal{B} \otimes \Omega_{1}(\mathcal{H})$ of $(\Omega(\mathcal{B}) \otimes \Omega(\mathcal{H}))_{1}$ and the $\operatorname{map}_{\mathrm{id}_{\mathcal{B}}} \otimes \epsilon \otimes \operatorname{id}_{\mathcal{H}}: \mathcal{B} \otimes \Omega_{1}(\mathcal{H}) \rightarrow \mathcal{B} \otimes \mathcal{H}$. Thus, upon the identification of $\Gamma_{\text {inv }}$ and ker $\epsilon$, any Đurđevič's connection form is a connection form in the sense of Definition 7 , and vice-versa.

This observation implies that there must be a very close relationship between the two approaches to the theory of noncommutative algebraic principal bundles and their characteristic classes. However, there is still a very important point in which they disagree, which is the differential calculi on the considered objects. Even if the space $\Omega_{1}(\mathcal{M})=$ $\left(\Omega_{1}(\mathcal{B})\right)^{\operatorname{Co} \Omega(\mathcal{H})}$ is generated by $\mathcal{M}$, there is no guarantee that the whole algebra $\Omega(\mathcal{M})$, defined as the algebra of coinvariants, is generated by $\mathcal{M}$. Hence the range of the Weil homomorphism, the homology of $\Omega(\mathcal{M})$, is different in general.

The consequence of this conceptual difference is that the notion of a strong connection, which is one of important tools to work with Brzeziński-Majid approach to Hopf-Galois extensions, can hardly be translated into Đurđevič's language. For instance, if we take Đurđevič's connections and Đurđevič's definition of $\Omega(\mathcal{M})$, then the strongness condition (iii) is vacuous as soon as the Hopf algebra $\mathcal{H}$ is cosemisimple. This observation is a 
direct consequence of the following decomposition proven in [7] under the assumption of cosemisimplicity of the Hopf algebra:

$$
\Omega(\mathcal{M}) \otimes \mathcal{B} \leftrightarrow \mathfrak{h o r}(\mathcal{B}) \leftrightarrow \mathcal{B} \otimes \Omega(\mathcal{M})
$$

Here the maps $\Omega(\mathcal{M}) \otimes \mathcal{B} \rightarrow \mathfrak{h o r}(\mathcal{B})$ and $\mathcal{B} \otimes \Omega(\mathcal{M}) \rightarrow \mathfrak{h o r}(\mathcal{B})$ are given by the multiplication. Another fact that one should use here is that, for all $p \in \mathcal{B}$, the element $d p-p_{(0)} \omega\left(p_{(1)}\right)$ belongs to $\mathfrak{h o r}(\mathcal{B})$. (Remember that we assume the connection $\omega$ to satisfy Đurđevič's relations.)

2. The construction of characteristic classes. In what follows, we shall always suppose the differential calculus on the base $\mathcal{M}$ defined as proposed by Đurđevič. Now we present a new construction of characteristic classes for noncommutative algebraic principal bundles generalizing both the "universal construction" of Đurđevič [8] and the construction of the author [12]. Moreover, we hope that it combines the advantages of both approaches and relates the theory of noncommutative bundles to the theory of the cyclic cohomology of Hopf algebras, as described in [3].

M. Đurđevič's universal construction allows one to define for arbitrary (i.e., not necessarily regular) connections a series of characteristic classes with values in the cohomology of the differential algebra $\Omega(\mathcal{M})$. The main point of this construction is the domain of the corresponding "Weil homomorphism". Recall that, in the classical case, this homomorphism is defined on the algebra of $A d$-invariant functions on the Lie algebra. It is precisely this construction that one imitates for a regular connection, when it exists.

On the contrary, to deal with an arbitrary connection, one has to make use of the algebra of $\Omega(\mathcal{H})$-coinvariants in the universal differential calculus of the algebra $\Gamma_{i n v}^{\otimes}$. It is not difficult to show that the evident chain map $\Omega_{\text {univ }}\left(\Gamma^{\otimes_{i n v}}\right) \rightarrow \Omega(\mathcal{B})$ determined by a connection $\omega$ intertwines the coaction of $\Omega(\mathcal{H})$, and hence sends the aforesaid coinvariants to coinvariants on the right hand side, i.e., to the differential graded subalgebra $\Omega(\mathcal{M})$ of $\Omega(\mathcal{B})$. Moreover, it is shown in [8] that the image of the corresponding map in homology does not depend on the choice of a connection. It is now natural to call this map the Weil homomorphism and the classes in its image characteristic classes.

As far as the second construction mentioned above is concerned, it arose from an attempt to answer the question whether there exists at least one regular connection for a given choice of differential calculi. It turns out that there is a special cochain complex and a uniquely defined class in its cohomology such that the existence of regular connections is equivalent to the statement that this class vanishes.

The complex employed in [12] is very hard to understand. However, it is possible to simplify its construction and map it to the Hochschild complex of the algebra $\Omega(\mathcal{M})$ considered just as a graded algebra. Of course, it is not correct to think that the class described above vanishes if its image in the Hochschild cohomology vanishes, but the contrary is true, and it is often enough to consider this image to show that there exist no regular connections.

This construction gives rise to still another variant of the Weil homomorphism. It is defined on the subalgebra of ad-invariant elements in $\Gamma_{i n v}^{\otimes}$ and takes values in the Hochschild cochain complex of $\Omega(\mathcal{M})$. 
Below we give a construction of the Weil homomorphism defined on the same algebra as in Đurđevič's construction (its close relative), but taking values in the Hochschild cohomology of the differential graded algebra $\Omega(\mathcal{M})$. The fact that this time the differential structure on $\Omega(\mathcal{M})$ comes into play is the main feature distinguishing our construction here from that of [12]. Before we proceed, let us recall once again that the differential structures that we choose on $\mathcal{B}$ and $\mathcal{H}$ are the universal ones, even though most of the following ideas can be reformulated for a more general case.

First of all we shall describe an important differential graded algebra, which will play a crucial role in further developments. In fact, this algebra has already appeared in a more or less explicit form in [8] and [3]. The definitions given in the cited papers are slightly different and here we chose the variant that better fits our purposes.

Definition 8. Let $\mathcal{H}$ be a Hopf algebra. Then the (reduced) noncommutative Weil complex of $\mathcal{H}$ is the free (tensor) graded algebra generated by the elements $1, i_{h}$ and $w_{h}$, where $h \in \operatorname{ker} \epsilon, \operatorname{deg} 1=0, \operatorname{deg} i_{h}=1$ and $\operatorname{deg} w_{h}=2$, with the differential defined by the formulas

$$
\begin{aligned}
\partial 1 & =0 \\
\partial i_{h} & =w_{h}-\sum_{h} i_{\bar{h}_{(1)}} \otimes i_{\bar{h}_{(2)}}, \\
\partial w_{h} & =\sum_{h}\left\{i_{\bar{h}_{(1)}} \otimes w_{\bar{h}_{(2)}}-w_{\bar{h}_{(1)}} \otimes i_{\bar{h}_{(2)}}\right\} .
\end{aligned}
$$

Here and below $\bar{h}$ denotes the image of the elemet $h \in \mathcal{H}$ under the canonical projection to $\operatorname{ker} \epsilon: \bar{h}=h-\epsilon(h) \cdot 1$. In particular, $\sum_{h} \bar{h}_{(1)} \otimes \bar{h}_{(2)}$ is the image in ker $\epsilon^{\otimes 2}$ of the coproduct of the element $h$. Note that this combination of the coproduct and projection is coassociative. (We did not write $\bar{h}$ in the l.h.s. of (18) and (19), because it was assumed above that $h \in \operatorname{ker} \epsilon$.) We shall denote this complex by $W(\widetilde{\mathcal{H}})$.

A simple calculation shows that $\partial^{2}=0$. Moreover, it is easy to show that this complex is acyclic. In fact, up to a change of grading it is isomorphic to the differential graded algebra $\Omega_{\text {univ }}\left(\operatorname{ker} \epsilon^{\otimes}\right)$. To see this, it is enough to consider the chain map $\eta: \Omega_{\text {univ }}\left(\operatorname{ker} \epsilon^{\otimes}\right) \rightarrow W(\widetilde{\mathcal{H}})$ that sends an element $h \in \operatorname{ker} \epsilon$ to $i_{h}$, and extends to the whole $\Omega_{\text {univ }}\left(\operatorname{ker} \epsilon^{\otimes}\right)$ with the help of the universal properties of the algebras in question. The inverse of this map is given by $\eta^{-1}\left(i_{h}\right)=h, \eta^{-1}\left(w_{h}\right)=d h+\sum \bar{h}_{(1)} \otimes \bar{h}_{(2)}$.

In order to explain this change of grading, observe that the tensor algebra $\operatorname{ker} \epsilon^{\otimes}$ is isomorphic to the algebra of left $\mathcal{H}$-coinvariant elements in $\Omega(\mathcal{H})$, which is the free algebra generated by its degree 1 part, $\Gamma_{i n v} \cong \operatorname{ker} \epsilon$. If we introduce on $\Omega_{u n i v}\left(\Gamma_{i n v}^{\otimes}\right)$ the grading equal to the sum of two natural gradings on it, then the isomorphism $\eta$ will be grading preserving.

Thus the above construction becomes a particular case of the complex in Đurdevič's "universal construction" when one chooses the universal differential calculus on the structure quantum group. On the other hand, it is just a variation of the definition in [3], where almost the same complex is defined, save that the set of generators there is indexed by the whole Hopf algebra $\mathcal{H}$ rather than by $\operatorname{ker} \epsilon$, as we do in this paper.

Now, observe that because of the identification $W(\widetilde{\mathcal{H}})=\Omega_{\text {univ }}\left(\Gamma_{\text {inv }}^{\otimes}\right)$, one can define 
the right $\Omega(\mathcal{H})$-coaction on the reduced Weil complex. It is easy to check that this coaction is determined by the following explicit formulae on the generating elements:

$$
\begin{aligned}
\widehat{F}\left(i_{h}\right) & =\sum i_{\bar{h}_{(2)}} \otimes S\left(h_{(1)}\right) h_{(3)}+1 \otimes \pi(h), \\
\widehat{F}\left(w_{h}\right) & =\sum w_{\bar{h}_{(2)}} \otimes S\left(h_{(1)}\right) h_{(3)} .
\end{aligned}
$$

Here $\pi(h)=\sum S\left(h_{(1)}\right) d h_{(2)}$, see above.

Let $I(\widetilde{\mathcal{H}})$ denote the subalgebra of $\Omega(\mathcal{H})$-coinvariant elements in $W(\widetilde{\mathcal{H}})$. It is this subalgebra that serves as the source of the generalized Weil homomorphism in Đurđevič's universal construction. Now we shall generalize it to obtain a map to the Hochschild complex.

To this end, let $C H^{\bullet}(\Omega(\mathcal{M}), \Omega(\mathcal{B}))$ be the Hochschild cohomology complex of the differential graded algebra $\Omega(\mathcal{M})$ with values in the $\Omega(\mathcal{M})$-bimodule $\Omega(\mathcal{B})$. Recall that

$$
C H^{\bullet}(\Omega(\mathcal{M}), \Omega(\mathcal{B}))=\bigoplus_{n \geq 0} \operatorname{Hom}\left(\Omega(\mathcal{M})^{\otimes n}, \Omega(\mathcal{B})\right)
$$

Here Hom denotes the space of $\mathbb{C}$-linear maps.

The grading on this complex is given by the usual rule: a map $\varphi: \Omega(\mathcal{M})^{\otimes n} \rightarrow$ $\Omega(\mathcal{B})$ changing the degree by $l \in \mathbb{Z}$ is assigned the degree $l+n$. The differential in $C H^{\bullet}(\Omega(\mathcal{M}), \Omega(\mathcal{B}))$ is defined as follows. Let $\varphi$ be as above, then

$$
\delta \varphi=\delta_{I} \varphi+\delta_{I I} \varphi
$$

where for $\delta_{I} \varphi: \Omega(\mathcal{M})^{\otimes n} \rightarrow \Omega(\mathcal{B})$ and $\delta_{I I} \varphi: \Omega(\mathcal{M})^{\otimes n+1} \rightarrow \Omega(\mathcal{B})$ we have

$$
\begin{aligned}
\left(\delta_{I} \varphi\right)\left(a_{1}, \ldots, a_{n}\right)= & d \varphi\left(a_{1}, a_{2}, \ldots, a_{n}\right) \\
& +\sum_{i=1}^{n}(-1)^{l \varepsilon_{i-1}} \varphi\left(a_{1}, \ldots, a_{i-1}, d a_{i}, a_{i+1}, \ldots, a_{n}\right), \\
\left(\delta_{I I} \varphi\right)\left(a_{1}, \ldots, a_{n+1}\right)= & a_{1} \varphi\left(a_{2}, \ldots, a_{n+1}\right) \\
+ & \sum_{i=1}^{n}(-1)^{l \varepsilon_{i-1}} \varphi\left(a_{1}, \ldots, a_{i-1}, a_{i} a_{i+1}, a_{i+2}, \ldots, a_{n+1}\right) \\
+ & (-1)^{l \varepsilon_{n}} \varphi\left(a_{1}, \ldots, a_{n}\right) a_{n+1}, \quad \varepsilon_{i}:=\left|a_{1}\right|+\ldots+\left|a_{i}\right|-i .
\end{aligned}
$$

Finally, recall that the Hochschild cohomology complex of an algebra with values in another algebra is an algebra itself, with multiplication (the cup-product) of $\varphi: \Omega(\mathcal{M})^{\otimes n} \rightarrow$ $\Omega(\mathcal{B})$ as above and $\psi: \Omega(\mathcal{M})^{\otimes m} \rightarrow \Omega(\mathcal{B})$ given by

$$
(\psi \cup \varphi)\left(a_{1}, \ldots, a_{m+n}\right)=(-1)^{l \varepsilon_{m}} \psi\left(a_{1}, \ldots, a_{m}\right) \varphi\left(a_{m+1}, \ldots, a_{m+n}\right) .
$$

In addition, since $\Omega(\mathcal{B})$ is an $\Omega(\mathcal{H})$-comodule, we can define an $\Omega(\mathcal{H})$-coaction on this complex simply by letting it coact on the image of a map.

Now we can define the chain map $\Phi: W(\widetilde{\mathcal{H}}) \rightarrow C H^{\bullet}(\Omega(\mathcal{M}), \Omega(\mathcal{B}))$ just by defining it on the (free) generators of $W(\widetilde{\mathcal{H}})$. Let $\omega$ be a (Đurđevič) connection and $R_{\omega}$ its curvature. We put

$$
\begin{aligned}
\Phi\left(i_{h}\right) & =\omega(\pi(h)), \\
\Phi\left(w_{h}\right) & =R_{\omega}(\pi(h))-[\cdot, \omega(\pi(h))] .
\end{aligned}
$$


Here $\omega(\pi(h))$ and $R_{\omega}(\pi(h))$ are regarded as the elements of

$$
\Omega(\mathcal{M})=\operatorname{Hom}(\mathbb{C}, \Omega(\mathcal{B})) \subset C H^{\bullet}(\Omega(\mathcal{M}), \Omega(\mathcal{B})),
$$

while the second term in $(28)$ is the commutator of a differential form with $\omega(\pi(h))$. Clearly, $[\cdot, \omega(\pi(h))] \in \operatorname{Hom}(\Omega(\mathcal{M}), \Omega(\mathcal{B})) \subset C H^{\bullet}(\Omega(\mathcal{M}), \Omega(\mathcal{B}))$. Observe that, due to the grading introduced above, the right hand side of (27) has degree 1 , while both terms in the r.h.s. of the formula (28) are of degree 2. Hence the map $\Phi$ is grading preserving. The following proposition is the main result of this paper.

Proposition 3. The map $\Phi$ commutes with the differentials and with the $\Omega(\mathcal{H})$ coaction, and its restriction $\widetilde{\Phi}$ to the subalgebra $I(\widetilde{\mathcal{H}})$ does not depend (up to a chain homotopy) on the choice of a (Đurđevič) connection.

Proof. To prove the first statement, it is enough to check it for the generating elements $i_{h}$ and $w_{h}$. This is done by direct calculations with formulas (23)-(25). In fact, it is enough to prove that

$$
\delta \Phi\left(i_{h}\right)=\Phi\left(\partial i_{h}\right)=\Phi\left(w_{h}-\sum i_{\bar{h}_{(1)}} \otimes i_{\bar{h}_{(2)}}\right) .
$$

Indeed, it then follows from the definition of $\Phi$ that $\Phi\left(w_{h}\right)=\delta \Phi\left(i_{h}\right)+\sum \Phi\left(i_{\bar{h}_{(1)}}\right) \cup \Phi\left(i_{\bar{h}_{(2)}}\right)$. Hence

$$
\begin{aligned}
\delta \Phi\left(w_{h}\right) & =\sum\left(\delta \Phi\left(i_{\bar{h}_{(1)}}\right) \cup \Phi\left(i_{\bar{h}_{(2)}}\right)-\Phi\left(i_{\bar{h}_{(1)}}\right) \cup \delta \Phi\left(i_{\bar{h}_{(2)}}\right)\right) \\
& =\sum\left(\Phi\left(\partial i_{\bar{h}_{(1)}}\right) \cup \Phi\left(i_{\bar{h}_{(2)}}\right)-\Phi\left(i_{\bar{h}_{(1)}}\right) \cup \Phi\left(\partial i_{\bar{h}_{(2)}}\right)\right) \\
& =\sum\left(\Phi\left(i_{\bar{h}_{(1)}}\right) \cup \Phi\left(w_{\bar{h}_{(2)}}\right)-\Phi\left(w_{\bar{h}_{(1)}}\right) \cup \Phi\left(i_{\bar{h}_{(2)}}\right)\right) \\
& =\Phi\left(\partial w_{h}\right)
\end{aligned}
$$

because the terms with $\sum \Phi\left(i_{\bar{h}_{(1)}}\right) \cup \Phi\left(i_{\bar{h}_{(2)}}\right) \cup \Phi\left(i_{\bar{h}_{(3)}}\right)$ cancel. Now to show that (29) holds, we compute:

$$
\begin{aligned}
\delta \Phi\left(i_{h}\right) & =\delta(\omega(\pi(h)))=\delta_{I}(\omega(\pi(h)))+\delta_{I I}(\omega(\pi(h))) \\
& =d \omega(\pi(h))+[\cdot, \omega(\pi(h))] \\
& =R_{\omega}(\pi(h))+\sum \omega\left(\pi\left(h_{(1)}\right)\right) \omega\left(\pi\left(h_{(2)}\right)\right)+[\cdot, \omega(\pi(h))] \\
& =\Phi\left(w_{h}\right)-\sum \Phi\left(i_{\bar{h}_{(1)}}\right) \cup \Phi\left(i_{\bar{h}_{(2)}}\right) .
\end{aligned}
$$

Here we have used the definition of $R_{\omega}$, see (15). The fact, that the map $\Phi$ commutes with the coaction is a direct consequence of the equations (20)-(21) and the definition of a Đurđevič connection. Note that now we can conclude that $\Phi$ indeed maps the subalgebra $I(\widetilde{\mathcal{H}})$ to the Hochschild complex of the graded algebra $\Omega(\mathcal{M}), C H^{\bullet}(\Omega(\mathcal{M}), \Omega(\mathcal{M})) \subseteq$ $C H^{\bullet}(\Omega(\mathcal{M}), \Omega(\mathcal{B}))$.

The second statement of this proposition is proved by a usual argument, see, e.g., [8]. Namely, let $\omega_{1}$ and $\omega_{2}$ be two connections and let $\Phi_{1}$ and $\Phi_{2}$ be the corresponding characteristic maps. Then for any $0 \leq t \leq 1$, the pseudotensorial form $\omega(t) \stackrel{\text { def }}{=} t \omega_{1}+(1-$ t) $\omega_{2}$ is a connection too. Now, we can consider this connection form as a map from $\Gamma_{i n v}$ to the differential graded algebra $\Omega\left(\mathcal{B} \times \mathbb{R}^{1}\right) \stackrel{\text { def }}{=} \Omega(\mathcal{B}) \otimes \Omega\left(\mathbb{R}^{1}\right)$, where $\Omega\left(\mathbb{R}^{1}\right)=\mathbb{R}[t] \otimes \Lambda[d t]$ is the complex of polynomial differential forms on $\mathbb{R}^{1}$. Observe that $\Omega(\mathcal{H})$ coacts on this algebra from the right, and that $\omega(t)$ satisfies the usual relations of a connection 
with respect to this coaction. Now we can define the characteristic map $\Phi_{t}: W(\widetilde{\mathcal{H}}) \rightarrow$ $C H^{\bullet}\left(\Omega(\mathcal{M}), \Omega(\mathcal{B})_{t}\right)$ by the usual formula. Finally, the integration of $\Phi_{t}$ from 0 to 1 gives the desired homotopy between $\Phi_{1}$ and $\Phi_{2}$.

In fact, the first part of the proposition is just a consequence of the isomorphism $\eta: \Omega_{\text {univ }}\left(\Gamma_{i n v}{ }^{\otimes}\right) \rightarrow W(\widetilde{\mathcal{H}})$, and the fact that the former differential graded algebra is (freely) generated by $\pi(h), h \in \Gamma_{i n v}$. Hence, one can define the map from this algebra to the differential graded algebra $C H^{\bullet}(\Omega(\mathcal{M}), \Omega(\mathcal{B}))$ by setting it on the generators as $\pi(h) \mapsto \omega(\pi(h))$, and then extending to the whole algebra so as to obtain a homomorphism of differential graded algebras. Thus, our construction is a rather simple generalization of the Đurđevič one. More precisely, if we substitute $C H^{\bullet}(\Omega(\mathcal{M}), \Omega(\mathcal{B}))$ for its subalgebra $\Omega(\mathcal{B})$ and put $i_{h} \mapsto \omega(\pi(h)), w_{h} \mapsto R_{\omega}(\pi(h))$, we obtain Đurđevič's universal map.

On the other hand, let us consider the quotient algebra of $W(\widetilde{\mathcal{H}})$ by the ideal generated by the elements $i_{h}$. One can regard it as the free algebra with generators $w_{h}$, and zero differential. Now, if we put $w_{h} \mapsto[\cdot, \omega(\pi(h))]$, we shall obtain (after passing to coinvariant elements) the map considered in [12].

However, as shown in [8], the homology of the universal algebra $I(\widetilde{\mathcal{H}})$ is trivial. Therefore, in order to obtain non-trivial classes, one should either consider a non-universal differential calculus and the corresponding space of coinvariants in $\Omega_{u n i v}\left(\Gamma_{i n v}{ }^{\otimes}\right.$ ) (one easily checks that it is possible to repeat the whole construction in this case), or pass to cyclic-type complexes. We end this paper by a discussion of the possible ways to obtain similar maps with values in cyclic-type cohomology.

The most obvious way to do it is to consider the map

$$
\widetilde{\Phi}_{\sharp}: I(\widetilde{\mathcal{H}})_{\sharp} \stackrel{\text { def }}{=} I(\widetilde{\mathcal{H}}) /[I(\widetilde{\mathcal{H}}), I(\widetilde{\mathcal{H}})] \rightarrow C H^{\bullet}(\Omega(\mathcal{M}), \Omega(\mathcal{M}))_{\sharp} \cdot
$$

The subscript $\sharp$ denotes the factorization by the subspace generated by the graded commutators. Observe that

$$
C H^{\bullet}(\Omega(\mathcal{M}), \Omega(\mathcal{M}))_{\sharp} \subseteq \bigoplus_{n \geq 0} \operatorname{Hom}\left(\Omega(\mathcal{M})_{\lambda}^{\otimes n}, \Omega(\mathcal{M})_{\sharp}\right) .
$$

Here $\Omega(\mathcal{M})_{\lambda}^{\otimes n}$ denotes the space of cyclically-invariant elements in the $n$-th tensor power of $\Omega(\mathcal{M})$. Clearly, our proof of homotopy invariance can be word-by-word repeated in this case, giving the Weil homomorphism with values in the homology of $C H^{\bullet}(\Omega(\mathcal{M}), \Omega(\mathcal{M}))_{\sharp}$. If we restrict the map $\alpha: \Omega(\mathcal{M})_{\lambda}^{\otimes n} \rightarrow \Omega(\mathcal{M})_{\sharp}$ in the image of the Weil homomorphism to $\mathcal{M}_{\lambda}^{\otimes n}$ and compose it with a trace on $\Omega(\mathcal{M})$, we shall obtain an element (cocycle) in the cocyclic complex of the algebra $\mathcal{M}$.

On the other hand, one can pass to a similar quotient space already at the level of the (reduced) Weil complex. Then, although it is, of course, not true that $\Omega(\mathcal{H})$ coacts on $W(\widetilde{\mathcal{H}})_{\sharp}$, we still can consider the coaction of the corresponding quotient coalgebra $\Omega(\mathcal{H})_{\sharp}$. (It is easy to show, that the space of the graded commutators $[\Omega(\mathcal{H}), \Omega(\mathcal{H})]$ is a coideal.) Thus, we can consider the space of "cyclic coinvariants": $I_{\sharp}(\widetilde{\mathcal{H}}) \stackrel{\text { def }}{=} W(\widetilde{\mathcal{H}})_{\sharp} \operatorname{Co} \Omega(\mathcal{H})_{\sharp}$.

Similarly, we shall have to generalize the target of our map. The corresponding chain map $\Phi_{\sharp}$ will now take values in $C H^{\bullet}(\Omega(\mathcal{M}), \Omega(\mathcal{B}))_{\sharp}$ regarded as a subcomplex in $C_{\sharp}(\Omega(\mathcal{M}), \Omega(\mathcal{B})) \stackrel{\text { def }}{=} \bigoplus \operatorname{Hom}\left(\Omega(\mathcal{M})_{\lambda}^{\otimes n}, \Omega(\mathcal{B})_{\sharp}\right)$. The latter complex is coacted on the 
right by the same coalgebra $\Omega(\mathcal{H})_{\sharp}$, and the map $\Phi_{\sharp}$, clearly, intertwines the coaction. Thus, we conclude that the image of $I_{\sharp}(\widetilde{\mathcal{H}})$ is in the corresponding space of coinvariants, which one can regard as the space of those maps in $C_{\sharp}(\Omega(\mathcal{M}), \Omega(\mathcal{B}))$ that send $\Omega(\mathcal{M})_{\lambda}^{\otimes n}$ to the coinvariants $\Omega_{\sharp}(\mathcal{M}) \stackrel{\text { def }}{=} \Omega(\mathcal{B})_{\sharp}^{\text {Co } \Omega(\mathcal{H})_{\sharp}}$. This map we shall denote by $\widehat{\Phi}_{\sharp}$.

Much as before, we can restrict the cocycles $\alpha$ in the image of $\widehat{\Phi}_{\sharp}$ to the subspace $\mathcal{M}_{\lambda}^{\otimes n}$, and compose them with a trace on $\Omega_{\sharp}(\mathcal{M})$, thus obtaining an element in the cyclic cohomology of $\mathcal{M}$.

Note that $I(\widetilde{\mathcal{H}})_{\sharp} \subseteq I_{\sharp}(\widetilde{\mathcal{H}})$, and a similar inclusion holds for the ranges of $\widehat{\Phi}_{\sharp}$ and $\widetilde{\Phi}_{\sharp}$. Consequently, the latter construction is a generalized version of the former. We shall address the question of calculation of these characteristic classes in a forthcoming paper.

Finally, let us point out another interesting question connected with this construction. Namely, if $\mathcal{A}$ is an algebra acted on (from the left) by a Hopf algebra $\mathcal{H}$, one can form the crossed product $\mathcal{A} \rtimes \mathcal{H}$, which is, in an evident way, a Hopf-Galois extension of $\mathcal{A}$ by $\mathcal{H}$. Hence, one can apply the construction considered in this paper to this particular example of a noncommutative algebraic principal bundle to obtain characteristic classes.

For instance, one can choose the universal differential calculus on $\mathcal{A} \rtimes \mathcal{H}$ and $\mathcal{H}$ as the starting point. Then the resulting classes will belong to an appropriate (see above) bivariant cyclic homology of the resulting coinvariant algebra $\Omega(\mathcal{A})=\Omega(\mathcal{A} \rtimes \mathcal{H})^{\operatorname{Co} \Omega(\mathcal{H})}$. Restricting, as it is explained above, the classes in the image of the Weil homomorphism to $\mathcal{A}$ and taking composition with a trace on $\Omega(\mathcal{A})$, one gets classes in the cyclic cohomology of $\mathcal{A}$. It would be interesting to compare such classes with the classes defined by A. Connes, H. Moscovici and M. Crainic (see, e.g., [2] and [3]). Though it can be shown that $\mathcal{A}$-modules associated to the crossed product $\mathcal{A} \rtimes \mathcal{H}$ are free, their bimodule structure is far from trivial (it is regulated by the action of $\mathcal{H}$ involved in the definition), and one can hope that the classes defined above can be used to characterize this action.

Acknowledgements. The author would like to thank the organizers of the school/ conference Noncommutative Geometry and Quantum Groups in Warsaw for their hospitality, and to thank Dr. P. M. Hajac for many valuable suggestions concerning the contents and presentation of this paper.

\section{References}

[1] T. Brzeziński and S. Majid, Quantum group gauge theory on quantum spaces, Commun. Math. Phys. 157 (1993), 591-638. Erratum 167 (1995), 235.

[2] A. Connes and H. Moscovici, Hopf algebras, cyclic cohomology and the transverse index theorem, Commun. Math. Phys. 198 (1998), 199-246.

[3] M. Crainic, Cyclic cohomology of Hopf algebras, J. Pure Appl. Algebra 166 (2002), 29-66.

[4] J. Cuntz and D. Quillen, Algebra extensions and nonsingularity, J. Amer. Math. Soc. 8 (1995), 251-289.

[5] L. Dąbrowski, H. Grosse and P. M. Hajac, Strong connections and Chern-Connes pairing in the Hopf-Galois theory, Commun. Math. Phys. 220 (2001), 301-331.

[6] M. Đurđevič, Geometry of quantum principal bundles I, Commun. Math. Phys. 175 (1996), 457-520. 
[7] M. Đurđevič, Geometry of quantum principal bundles II, Rev. Math. Phys. 9 (1997), 531-603.

[8] M. Đurđevič, Characteristic classes of quantum principal bundles, Preprint, Institute of Mathematics, UNAM, Mexico (1995) q-alg/9507017.

[9] P. M. Hajac, Strong connections on quantum principal bundles, Commun. Math. Phys. 182 (1996), 579-617.

[10] M. Karoubi, Homologie cyclique et K-théorie, Astérisque 149 (1987) 147.

[11] H. F. Kreimer and M. Takeuchi, Hopf algebras and Galois extensions of an algebra, Indiana Univ. Math. J. 30 (1981), 675-692.

[12] G. I. Sharygin, Geometry of noncommutative principal bundles, Ph.D. thesis (in Russian). Submitted for publication to VINITI Math. Surveys.

[13] P. Schauenburg, Zur nichtkommutativen Differentialgeometrie von Hauptfaserbundeln Hopf-Galois-Erweiterungen von de Rham-Komplexen, Algebra Berichte 71, Verlag Reinhard Fischer, Munich, 1993.

[14] S. L. Woronowicz, Differential calculus on compact matrix pseudogroups (quantum groups), Commun. Math. Phys. 122 (1989), 125-170. 